\title{
Absorption-induced image resolution enhancement in scattering media
}

Mehbuba Tanzid, ${ }^{1,2}$ Nathaniel J. Hogan,, ${ }^{1,2}$ Ali Sobhani, ${ }^{1,2}$ Hossein Robatjazi, ${ }^{1,2}$ Adithya K.

Pediredla, ${ }^{1}$ Adam Samaniego, ${ }^{1}$ Ashok Veeraraghavan ${ }^{1}$ and Naomi J. Halas ${ }^{1,2}$

${ }^{1}$ Department of Electrical and Computer Engineering and ${ }^{2}$ Laboratory for Nanophotonics, Rice University, MS-366, 6100 Main Street, Houston, Texas 77005, United States

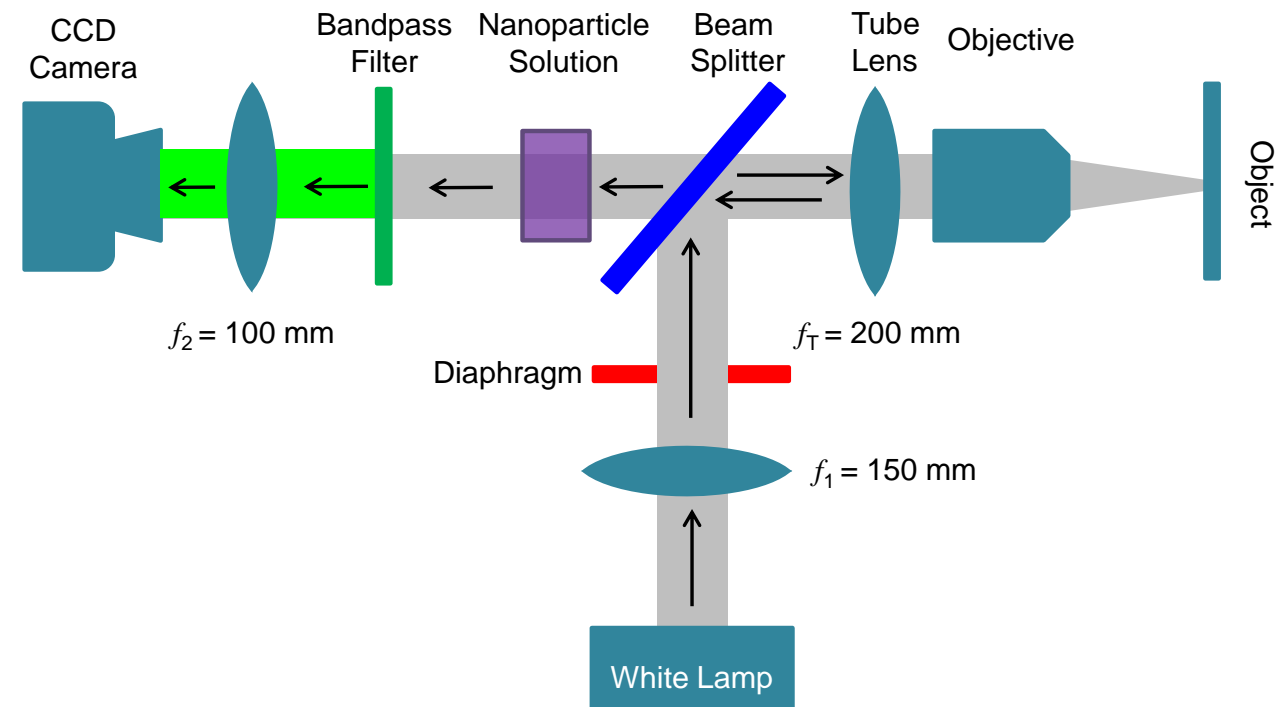

Supplementary Figure 1: Schematic top view of the imaging setup. Object to camera distance is $420 \mathrm{~mm}$ and lamp to beam splitter distance is $160 \mathrm{~mm}$.

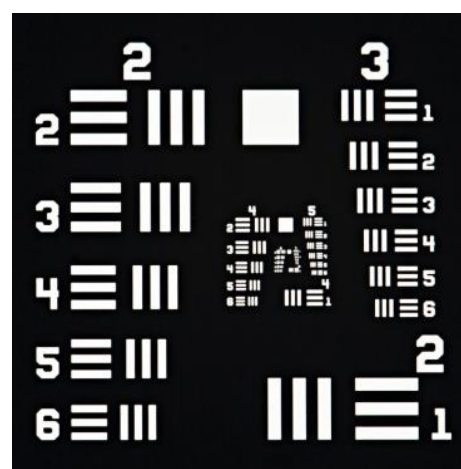

Supplementary Figure 2: 1951

USAF resolution target. 

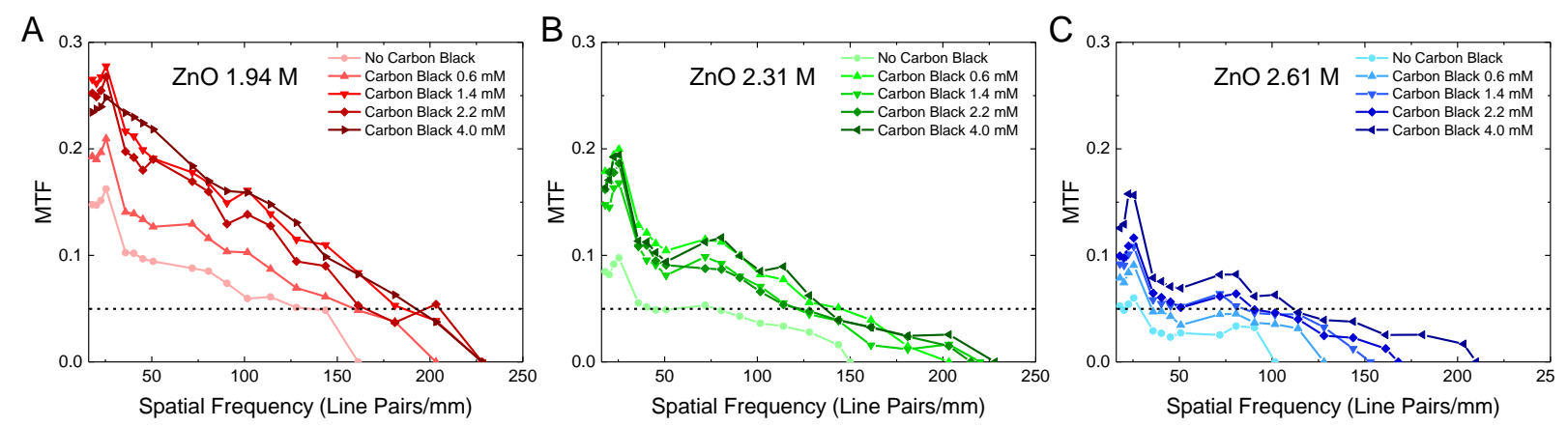

Supplementary Figure 3 Modulation transfer function (MTF) of ZnO and carbon black solutions. MTF of images obtained through mixture of carbon black and (A) $1.94 \mathrm{M}\left(\mu_{s}=11.4 \mathrm{~cm}^{-1}\right)$, (B) $2.31 \mathrm{M}\left(\mu_{s}=13.6 \mathrm{~cm}^{-1}\right)$, and $(\mathrm{C})$ $2.61 \mathrm{M}\left(\mu_{s}=15.4 \mathrm{~cm}^{-1}\right) \mathrm{ZnO}$ solutions. Dashed black line is drawn to obtain cut-off spatial frequency indicating $\mathrm{MTF}=0.05$ in each case.

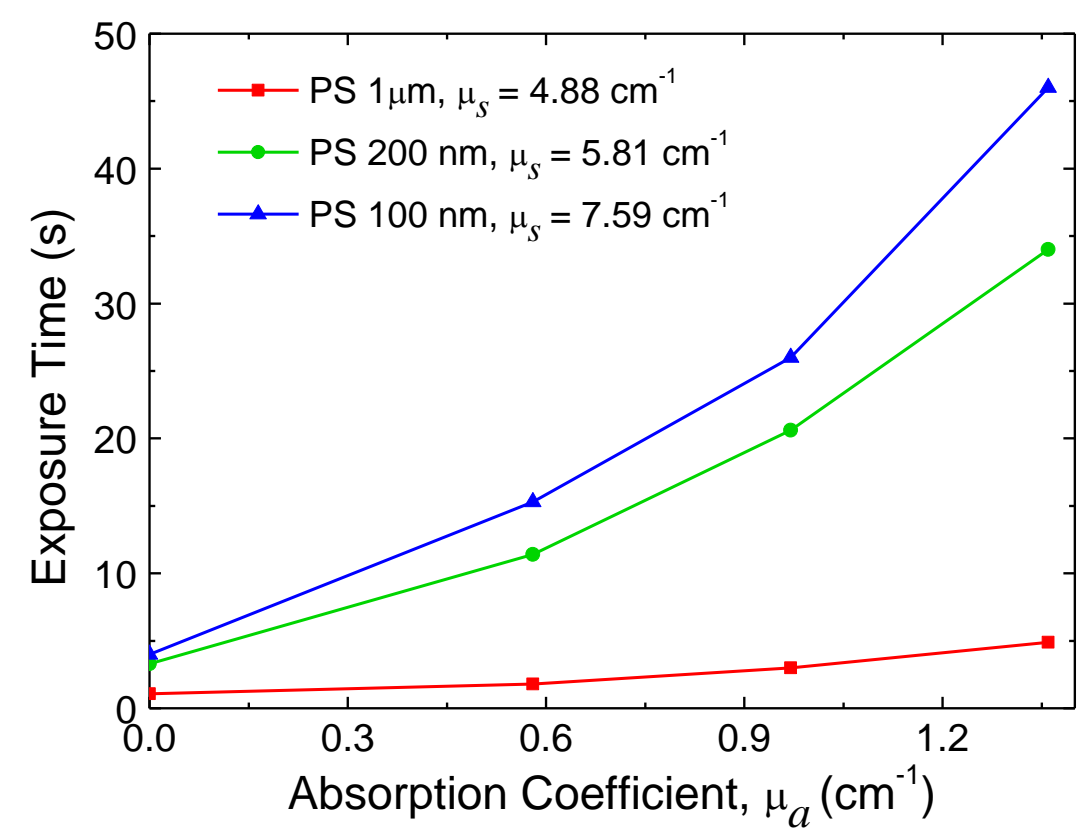

Supplementary Figure 4: Dependence of exposure time and absorption coefficient. 
A

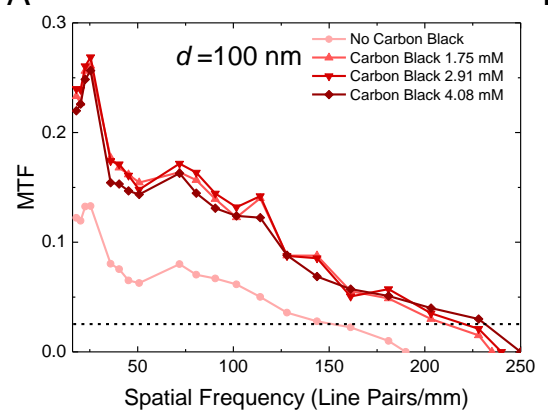

$\mathrm{B}$

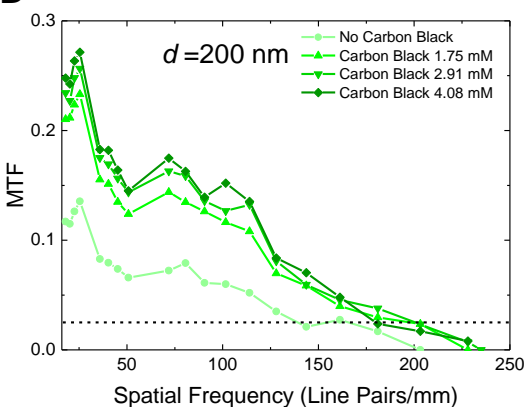

C

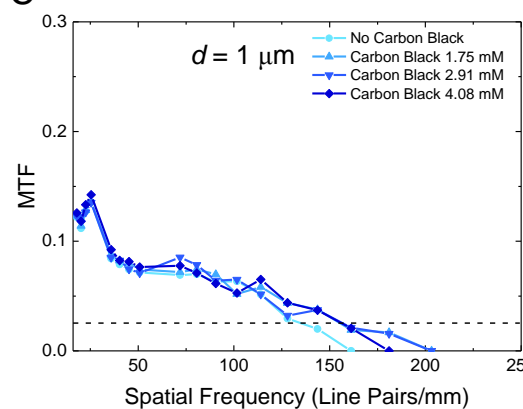

Supplementary Figure 5: Modulation transfer function (MTF) of polystyrene and carbon black solutions. MTF of images obtained through mixture of carbon black and (A) $100 \mathrm{~nm}$, (B) $200 \mathrm{~nm}$, and (C) $1 \mu \mathrm{m}$ diameter polystyrene solutions. Dashed black line indicates MTF $=0.05$ in each case.

Original

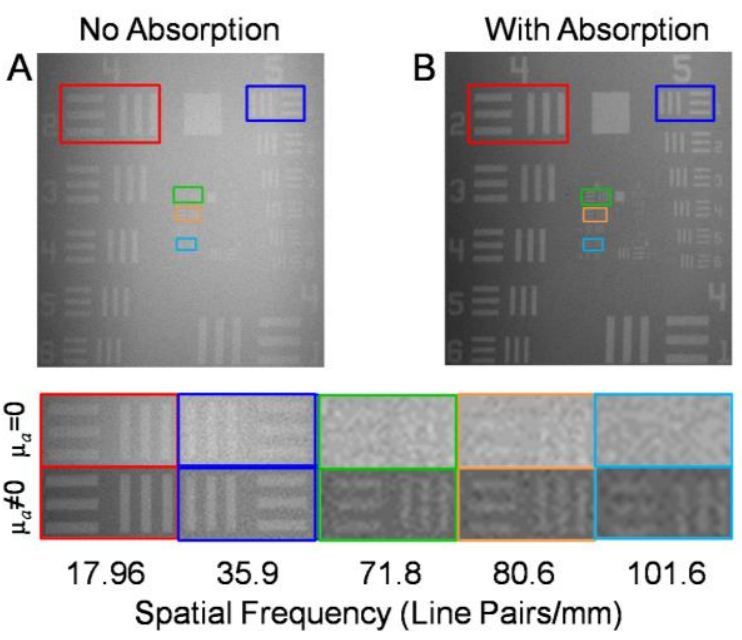

Contrast Enhanced

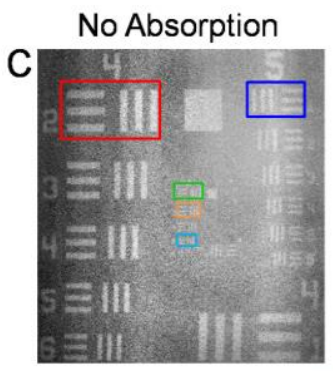

With Absorption
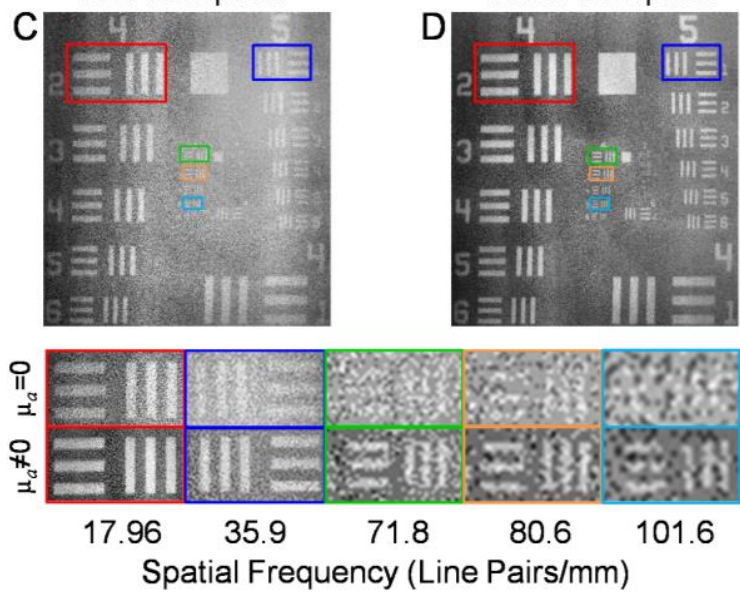

Supplementary Figure 6: Contrast enhancement. Original images obtained through $\mathrm{ZnO}$ as scattering medium with $\mu_{s}=15.4 \mathrm{~cm}^{-1}$ and $g=0.1$ with (A) no absorption and (B) $\mu_{s}=1.33 \mathrm{~cm}^{-1}$. Contrast enhanced images with (C) no absorption and (D) $\mu_{s}=1.33 \mathrm{~cm}^{-1}$. At the bottom showing zoomed in images of different spatial frequencies in each image. The absorption-induced resolution enhancement becomes more visible as the image contrast is enhanced. 


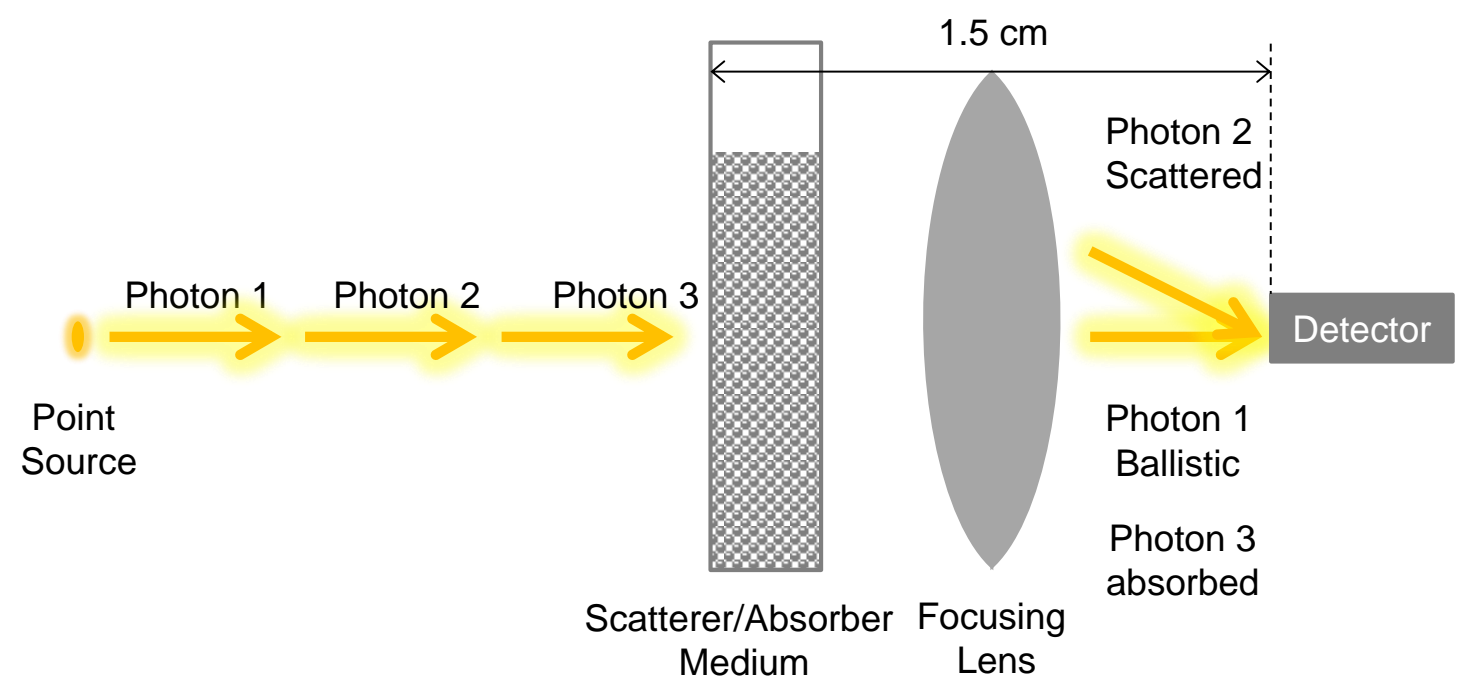

Supplementary Figure 7: Monte-Carlo simulation schematic setup.

\section{Henyey-Greenstein Scattering Phase Function:}

Henyey-Greenstein phase function mimics the angular dependence of light scattering by small particles which was empirically derived to model the scattering of light by intergalactic dust. It has been successfully applied for simulating various scattering media, such as oceans, clouds, and biological tissue. The function only depends on the scattering angle $\theta$ and is defined as following:

$$
p(\theta)=\frac{1-g^{2}}{4 \pi\left(1+g^{2}-2 g \cos \theta\right)^{3 / 2}}
$$

such that $\int_{0}^{\pi} p(\theta) 2 \pi \sin \theta d \theta=1$ and $\int_{0}^{\pi} \cos \theta p(\theta) 2 \pi \sin \theta d \theta=g$. Here $g \in[-1,1]$ is the anisotropy factor which determines the relative strength of forward and backward scattering. It represents the average cosine of the scattered directions or $\langle\cos \theta\rangle$. Positive values of $g$ give forward scattering, and negative values give backward scattering. The value of $g=0$ will give isotropic scattering. 


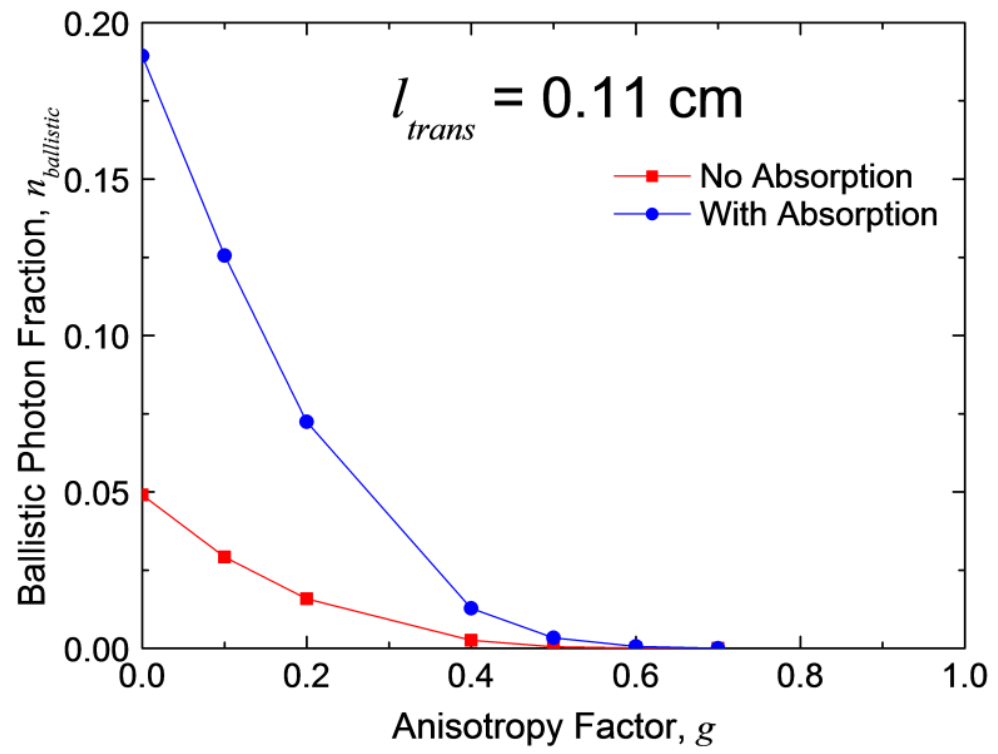

Supplementary Figure 8: Comparison of absorption-induced image enhancement for scattering media with equal transport mean free path $\left(l_{\text {trans }}=0.11 \mathrm{~cm}\right)$ but varying anisotropy factor. Ballistic photon fraction as a function of anisotropy factor, $g$ from Monte Carlo simulations of a scattering medium with no absorption (red) and with absorption coefficient $\mu_{a}=3.6 \mathrm{~cm}^{-1}$ (blue). Increase in $n_{\text {ballistic }}$ becomes negligible as $g$ reaches towards 1 and the medium becomes forward-scattering. 